\title{
Complementary Local and Nonlocal Measurements of Conjugate Transformation - Unified Logic Representation of Conjugate 0-1 Vectors
}

Jeffrey Zheng ( $\square$ conjugatelogic@yahoo.com )

Yunnan University

\section{Research Article}

Keywords: Conjugate Logic, Conjugate Transformation Structure, Phase Space, Selected Set, Complementary Set, Measure, Complementary Measure

Posted Date: September 16th, 2020

DOI: https://doi.org/10.21203/rs.3.rs-76524/v1

License: (a) (i) This work is licensed under a Creative Commons Attribution 4.0 International License.

Read Full License 


\title{
Complementary Local and Nonlocal Measurements of Conjugate Transformation - Unified Logic Representation of Conjugate 0-1 Vectors
}

Jeffrey Zheng

\begin{abstract}
Bohr proposed the complementarity principle in 1927 as the foundation of quantum mechanics, since then relevant debates have been critically discussed for many years. Applying a pair of spin particles, Einstein proposed the EPR paradox in 1935. Using nonlocal potential properties, Aharonov and Bohm proposed the $\mathrm{AB}$ effect to test complementary measurement results. Under locality conditions, Bell established a Bell inequality under classical logic. Using a pair of particles and double sets of ZMI devices for complementarity measurements, Hardy proposed the Hardy paradox in 1992. During the past 50 years, locality and nonlocality tests on complementarity were hot-topics among the advanced quantum information, computing and measurement directions with various theoretical extensions and solid experimental results.

These complementarity approaches separated local/nonlocal parameters to form different equations without an integrated logic framework to describe these equations including both local and nonlocal features consistently. The main results provide a series of paradoxes that conflict with each other.

This paper uses conjugate transformation. Based on the $m+1$ kernel form of $0-1$ states, $n$ pairs of conjugate partitions were established. Under a given configuration in $N$ bits, a set of $2 n 0-1$ feature vectors are applied to construct conjugate transformation operators in logic levels with intrinsic measurements to be a set of measurement operators.

The key results of the paper are listed in Theorem 5. Two special functions of vector logic (CNF or DNF expression) and four equivalent expressions of the elementary equation are examples to show local and nonlocal variables in equations consis-
\end{abstract}

Jeffrey Zheng

Yunnan University

Key Laboratory of Quantum Information of Yunnan

Key Laboratory of Software Engineering of Yunnan

School of Software e-mail: conjugatelogic@yahoo.com

Funding support: Core Technologies in Quantum Communication, Key Project of Yunnan Science and Technology (2018ZI002) 
tently. Applying two pairs of conjugate sets $\langle A, B\rangle$ and their complementary sets $\left\langle A^{\prime}, B^{\prime}\right\rangle, 4$ meta measures are established corresponding to $\left\{ \pm a_{A}, \pm b_{B}\right\}$ quantitative features under measurement operators. The main results of the paper are represented in Lemma (1-4), Theorem (1-5) and Corollary (1-7). From a vector logic viewpoint, conjugate complementary scheme can organize local and nonlocal variables to satisfy the comprehensive properties of modern logic constructions on completeness, non-conflict and consistence in a united logic framework.

Keywords Conjugate Logic, Conjugate Transformation Structure, Phase Space, Selected Set, Complementary Set, Measure, Complementary Measure

\section{Introduction}

\subsection{Complementarity Principle}

The origin of the complementarity principle [1] could be traced back to 1927 , Heisenberg proposed the uncertainty relation and Bohr proposed the complementarity principle for the foundation of quantum mechanics. In two Solvay conferences of 1927 and 1930, Einstein and Bohr started the strangest debate in the history of the understanding of the foundation of quantum mechanics [2]. Bohr used complementarity to explain wave-particle duality to provide the groundwork for the Copenhagen interpretation of quantum mechanics.

Using a pair of spin particles, Einstein proposed the EPR paradox [3] in 1935 and Bohr's reply [4]. Both quantum founders debated this issue for many years, until both masters were passed away, and there was no a common agreement achieved.

\subsection{Complementarity Measurements}

From a complementarity measurement viewpoint, Bohm proposed a hidden variable theory for quantum mechanics in 1952. Aharonov and Bohm proposed theoretical model and testing methods to use magnetic potentials in a double path interference device on the $\mathrm{AB}$ effect.

Based on the $\mathrm{AB}$ effect, Aharonov expanded to apply complementarity schemes to handle local and nonlocal attributes of topology [7]-[10]. Applying the Schroedinger equation, the measurement equation established with both $\delta$ and $\delta^{\prime}$ complementary variables [11]. Using differential equations to describe various complementary properties, there are many research results to describe wave-particle duality in various measurement models to show multiple interactive effects. 


\subsection{Bell Inequality}

Under locality conditions, Bell proposed the Bell inequality [12, 13] in 1964 using classical logic. Based on Bell's results, Clauser-Horne-Shimony-Holt CHSH proposed the CHSH inequality [14] in 1969, consequently relevant experiments tested. In 1989, Greenberger-Horne-Zeilinger GHZ proposed the GHZ theorem [15] to expand quantum interaction effects to break locality conditions in quantum systems by more than three Qubits [16]-[21].

\subsection{Hardy Paradox}

Bell inequality uses statistics of joint measurements. With a logical quantum-versusclassical contradiction without the need for inequality, the Hardy paradox provides a more striking conflict than that with inequalities. It is considered as "the simplest version of Bell theorem" and "one of the strangest and most beautiful gems yet to be found in the extraordinary soil of quantum mechanics" [24].

From 2009, a series of experiments [26]-[29] showed that this type of paradox existed in reality. Based on the Hardy paradox and extensions, various theoretical models and experiments were developed [26]-[31], linked with wider applications: Bell inequality, quantum entanglements, single photon detection etc.

From an algebraic viewpoint, Hardy paradox can be briefly described as $\left\langle e^{-}, e^{+}\right\rangle \rightarrow$ $\left\langle C^{-}, C^{+} \mid D^{-}, D^{+}\right\rangle$, where two pairs of elements $\left\langle e^{-}, e^{+}\right\rangle$in interactions as an input resource, two sets of double path interference devices are used to generate two groups of two pairs of four complementary measurements $\left\langle C^{-}, C^{+} \mid D^{-}, D^{+}\right\rangle$as a set of output results.

In the paper [29], $n$ variables of qubit system describes its index set as $I_{n}=$ $\{1,2, \cdots, n\}$. For the $k$-th Qubit, its projection set is labeled by $\left\{a_{k}, \bar{a}_{k}, b_{k}, \bar{b}_{k}\right\}$ as complementary measurements.

For $a, b \in\{0,1\}$ and $\bar{a}=\mathbf{1}-a, \bar{b}=\mathbf{1}-b, \alpha \subseteq I_{n}, \bar{a}=\prod_{k \in \alpha} \bar{a}_{k}$.

From a representing viewpoint, $\left\{a_{k}, \bar{a}_{k}, b_{k}, \bar{b}_{k}\right\}$ and $\left\{a_{\alpha}, \bar{a}_{\alpha}, b_{\alpha}, \bar{b}_{\alpha}\right\}$ provide equivalent information on two groups of two pairs of four measurements $\left\langle C^{-}, C^{+} \mid D^{-}, D^{+}\right\rangle$ in Hardy paradox.

\subsection{Weakness of Various Complementarity Frameworks}

From a logic viewpoint, listed schemes of complementarity have various weaknesses.

Studies on Bohr, Heisenberg, Einstein, Bohm and Aharonov have mainly applied advanced algebraic tools in mathematical physics such as matrix theory and differential equations. The principle of complementarity was based on classical logic at the concept level, and there is no any explicit logic equation associated with the 
discussed cases. Typical applications were merely focus on levels of thought experiments and weak measurements such as wave-particle duality, double split interferences, Schroedinger cat and a pair of spin particles etc.

Studies on Bell, CHSH, GHZ, Hardy, etc. use statistics of joint measurements in logic and probability equations in classical logic levels. There is a prelimitation on their expressions to put various complementary variables integrated into a logic equation to provide separate equations on either local or nonlocal forms respectively. The main targets provide distinguished paradoxes on various measurements.

In other words, the classical 0-1 logic system cannot provide consistent framework to support two groups of four complementary measurements with local and nonlocal properties in an equation without intrinsic contradiction. Such as Bell inequality and multiple experimental evidence on the Hardy paradox.

\subsection{Conjugate Transformation Structure}

Applying a balanced organization and pairs of partitions on phase spaces, Jeffrey Z.J. Zheng proposed conjugate classification, conjugate transformation [32, 33] and reversible logic equations [34] in the 1990s. In his PhD thesis [35], four regular plane lattices for binary images were applied for analysis and processing, and a conjugate transformation structure was established (the Section 5.4 of [35]). In this regard, the newest progress is a monograph published by Springer[36] in 2019 to propose a $0-1$ vector logic system from logic, measurement and visualization as key components for variant construction.

To make a proper logic foundation, this paper is initially from 0-1 logic through two basic vector logic functions and the elementary equation of the conjugate transformation to support Hardy variables consistently.

\subsection{Paper's Structure}

Based on the introduction in Section 1, in Section 2, basic logic constructions are described from variables, states, conjugate states and function spaces. In Section 3 , a sequence of state vectors is composed of configuration spaces. In Section 4, conjugate transformation is established by pairs of two sets of feature vectors to be a reversible logic expression. In Section 5, various operations on clusters are described for conjugate transformation. Measurement operators were illustrated in classical logic equations in DNF and CNF forms and four equivalent expressions of the elementary equation. In Section 6, four measurements are established to form probability spaces and in Section 7, the conclusion of the paper and future explorations are discussed. 


\section{Transforming Structure}

Complemetarity expressions are not restricted to measurement operators. In this paper, discrete vectors and transformation rules are provided to link with conjugate transformation with complementarity properties via various operators of different levels.

Conjugate logic construction is composed of hierarchical levels on $0-1$ vector structures. Using $m+1$ logic variables to make a kernel form, multiple invariants are proposed to partition pairs of vector states into various clusters to construct multiple levels of phase spaces.

Applying a pair of balanced foreground and background feature clusters, conjugate classification organizes multiple invariants to partition its phase spaces into $2 n$ classes. For a given configuration, it is possible to define a feature class to partition the relevant configuration as $2 n$ representatives to express this possible phase space consistently. Under transformation, $2 n$ classes are played in the most important role. Using this type of meta feature expression, it is feasible to establish an elementary equation of conjugate transformation.

\subsection{Kernel Form}

Let $K$ be a kernel form, it has a certain geometric-logic shape with $m+1$ vector logic variables: $K=\left\{x_{0}, x_{1}, \cdots, x_{k}, \cdots, x_{m}\right\}, x_{k} \in\{0,1\}, 0 \leq k \leq m$.

Let $x$ be a corresponding position of the kernel form $K, \vec{x}$ be a vector with $m+1$ elements and $\vec{x}=\left(x, x_{1}, \cdots, x_{k}, \cdots, x_{m}\right), K(\vec{x})$ be the kernel form of the $m+1$ elements.

For multiple variables, a kernel form has a special geometric structure and multiple geometric and topologic invariants, such as translation, reflection and rotation can be identified.

\subsection{Phase Spaces and Conjugate Phase Spaces}

Let $\Omega$ be a phase space - a state space of a kernel form $K(\vec{x})$. For a given $K(\vec{x})$, there are distinguished $m+1$ logic variables. For a given $x$, there are two values $(0,1)$, using this value as a reference, it is feasible to organize other $m$ variables to form a pair of two sets with conjugate vector states: $S(\vec{x})$ and its conjugation $\tilde{S}(\vec{x})$, $x_{k} \in\{0,1\}, 1 \leq k \leq m$.

$$
\begin{aligned}
& S(\vec{x})=K\left(x=1, x_{1}, \cdots, x_{k}, \cdots, x_{m}\right) \\
& \tilde{S}(\vec{x})=K\left(x=0, x_{1}, \cdots, x_{k}, \cdots, x_{m}\right)
\end{aligned}
$$


For convenience, let $\Theta$ and $\tilde{\Theta}$ express a pair of conjugate phase spaces associated with two set operators: $\{\wedge, \vee\}$,

$$
\begin{aligned}
\Theta & =\left\{S(\vec{x}) \mid x=1, x_{k} \in\{0,1\}, 1 \leq k \leq m\right\} \\
\tilde{\Theta} & =\left\{S(\vec{x}) \mid x=0, x_{k} \in\{0,1\}, 1 \leq k \leq m\right\} \\
\Omega & =\Theta \vee \tilde{\Theta} \\
\emptyset & =\Theta \wedge \tilde{\Theta}
\end{aligned}
$$

Let $|R|$ be the number of states in the set $R$.

Lemma 1. For a given $K(\vec{x})$ and its phase space $\Omega,|\Theta|=|\tilde{\Theta}|=2^{m}$,

$$
|\Omega|=|\Theta|+|\tilde{\Theta}|=2^{m+1}
$$

Two conjugate phase spaces partition the phase space in balance, and two conjugate phase spaces contain conjugate clusters as one to one pairs to organize all possible states as a balanced hierarchical structure.

\section{$2.32 n$ Feature Clusters of Conjugate Classification)}

Due to $\Theta$ and $\tilde{\Theta}$ with a pair of two vector state sets, each set contains $2^{m}$ states. Using a specific partition, it is feasible to define relevant clusters as $n$ groups. This classification is a conjugate classification if each group has a conjugate group and all groups are composed of the whole states.

Let $\Gamma_{j}\left(\tilde{\Gamma}_{j}\right)$ be the $j$-th cluster of $\Theta(\tilde{\Theta})$, for any state $S(\vec{x}) \in \Gamma_{j}$, iff, there is one corresponding state $\tilde{S}(\vec{x}) \in \tilde{\Gamma}_{j}, y=\neg x=0, y_{k}=\neg x_{k} ; x_{k}, y_{k} \in\{0,1\}, 0<k \leq m$, and vise versa.

Using a conjugate pair of 1-1 mapping, equations of $\left|\Gamma_{j}\right|=\left|\tilde{\Gamma}_{j}\right| \geq 1$ are true on the following equations.

$$
\begin{aligned}
\Theta & =\bigvee_{j=1}^{n} \Gamma_{j} \\
\tilde{\Theta} & =\bigvee_{j=1}^{n} \tilde{\Gamma}_{j} \\
\emptyset & =\Gamma_{k} \wedge \Gamma_{j}=\tilde{\Gamma}_{k} \wedge \Gamma_{j}=\Gamma_{k} \wedge \tilde{\Gamma}_{j}=\tilde{\Gamma}_{k} \wedge \tilde{\Gamma}_{j}, 1 \leq j \neq k \leq n
\end{aligned}
$$

Theorem 1. For a given conjugate classification, 2 feature classes $\left\{\left\{\Gamma_{j}\right\}_{j=1}^{n},\left\{\tilde{\Gamma}_{j}\right\}_{j=1}^{n}\right\}$ partition a pair of two conjugate phase spaces $\Theta$ and $\tilde{\Theta}$ respectively. 


\section{Configuration}

Let $X$ be a configuration that is composed of a sample set with $N$ elements, and each element is a state.

$$
\begin{aligned}
X & =\left(X_{1}, \cdots, X_{l}, \cdots, X_{N}\right), X_{l} \in \Omega .1 \leq l \leq N \\
X_{l} & =\left\{\begin{array}{l}
S\left(\overrightarrow{x^{l}}\right), X_{l} \in \Theta, \\
\tilde{S}\left(\overrightarrow{x^{l}}\right), X_{l} \in \tilde{\Theta} ;
\end{array}\right. \\
\overrightarrow{x^{l}} & =\left(x^{l}, x_{1}^{l}, \cdots, x_{k}^{l}, \cdots, x_{m}^{l}\right), x^{l}, x_{k}^{l} \in\{0,1\}, 1 \leq k \leq m
\end{aligned}
$$

From a representing viewpoint, $X$ may be equivalent to a $(m+1) \times N 0$-1 sequence to be composed of $N$ states, and each $X_{l}$ state contains $m+1$ bits.

i.e. When consequential states have overlapped parts in $m$ bits, $X$ could be represented as a $1 \mathrm{D}$ cellular automata with a total of $N$ cells and each cell with $m+1$ bits.

\subsection{Two Sets of Feature Vectors}

For convenient analysis, we suppose two states can be identified, iff they are in two distinguished groups. Under this condition, the configuration vector can be mapped into $2 n$ feature vectors.

Using defined $2 n$ classes, their feature values are:

$$
\begin{aligned}
& \Gamma_{j}\left(X_{l}\right)=\left\{\begin{array}{l}
1, X_{l} \in \Gamma_{j} ; \\
0, X_{l} \notin \Gamma_{j}
\end{array}\right. \\
& \tilde{\Gamma}_{j}\left(X_{l}\right)=\left\{\begin{array}{l}
0, X_{l} \in \tilde{\Gamma}_{j} \\
1, X_{l} \notin \tilde{\Gamma}_{j}
\end{array}\right.
\end{aligned}
$$

Under this paired mapping rule, all $X_{l}, 1 \leq l \leq N$ correspond to $2 n 0$ - 1 feature vectors.

$$
\begin{gathered}
\Gamma_{j}(X)=\left(\Gamma_{j}\left(X_{1}\right), \cdots, \Gamma_{j}\left(X_{l}\right), \cdots, \Gamma_{j}\left(X_{N}\right)\right), 1 \leq l \leq N, 1 \leq j \leq n \\
\tilde{\Gamma}_{j}(X)=\left(\tilde{\Gamma}_{j}\left(X_{1}\right), \cdots, \tilde{\Gamma}_{j}\left(X_{l}\right), \cdots, \tilde{\Gamma}_{j}\left(X_{N}\right)\right), 1 \leq l \leq N, 1 \leq j \leq n
\end{gathered}
$$

\subsection{Two Constant Vectors}

In addition to these feature vectors, there are two constant vectors. 


$$
\begin{aligned}
& \qquad \begin{array}{c}
\Gamma_{\emptyset}\left(X_{I}\right)=0,1 \leq l \leq N ; \\
\tilde{\Gamma_{\emptyset}}\left(X_{I}\right)=1,1 \leq l \leq N
\end{array} \\
& \text { Let two constant vectors be } \overrightarrow{0} \text { and } \overrightarrow{1}, \\
& \overrightarrow{0}=\Gamma_{\emptyset}(X)=\left(\Gamma_{\emptyset}\left(X_{1}\right) \cdots \Gamma_{\emptyset}\left(X_{l}\right) \cdots \Gamma_{\emptyset}\left(X_{N}\right)\right)=(0 \cdots 0 \cdots 0), 1 \leq l \leq N ; \\
& \overrightarrow{1}=\tilde{\Gamma_{\emptyset}}(X)=\left(\tilde{\Gamma_{\emptyset}}\left(X_{1}\right) \cdots \tilde{\Gamma_{\emptyset}}\left(X_{l}\right) \cdots \tilde{\Gamma_{\emptyset}}\left(X_{N}\right)\right)=(1 \cdots 1 \cdots 1), 1 \leq l \leq N
\end{aligned}
$$

Two constant vectors in configuration select $\emptyset$ feature groups.

\subsection{Feature Counting}

In a 1D cellular automata, the $l$-th position of feature vector $\Gamma_{j}(X)$ has a 1 value if $\left.X^{l} \in \Gamma_{j}\right\}$ and the $l$-th position of conjugate feature vector $\tilde{\Gamma}_{j}(X)$ has 0 values if $X^{l} \in \tilde{\Gamma}_{j}$. Both feature vectors are $0-1$ vectors with $N$ bits to be a feature projection from the configuration $X$ to be composed of two sets of $2 n$ feature vectors.

Counting the number of 1 elements in $\Gamma_{j}(X)$ and the number of 0 elements in $\tilde{\Gamma}_{j}(X)$, two coefficients $a_{j}, b_{j}$ can be calculated.

$$
\begin{aligned}
& a_{j}(X)=\sum_{l=1}^{N}\left(\Gamma_{j}\left(X_{l}\right)==1\right) \\
& b_{j}(X)=\sum_{l=1}^{N}\left(\tilde{\Gamma}_{j}\left(X_{l}\right)==0\right)
\end{aligned}
$$

In general, the feature vector length $N$ satisfies the following equation for $2 n$ coefficients.

$$
N=\sum_{j=1}^{n}\left(a_{j}(X)+b_{j}(X)\right)
$$

\section{Basic Logic Operations on Feature Vectors}

\subsection{Two Canonical Feature Vectors}

Using two logic operators $\{\cap, \cup\}$, combinations of whole feature vectors to be represented as two canonical feature vectors 


$$
\begin{gathered}
\Gamma(X)=\bigcup_{j=0}^{n} \Gamma_{j}(X) ; \\
\tilde{\Gamma}(X)=\bigcap_{j=0}^{n} \tilde{\Gamma}_{j}(X)
\end{gathered}
$$

Theorem 2. For a given configuration $X$, two canonical feature vectors satisfy $\Gamma(X)=\tilde{\Gamma}(X)$.

Proof. For a state in a feature group, its feature value is 1 and a state in a conjugate feature group, its special value is 0 . When two types of feature vectors are merged together only positions in feature groups keep values 1 under OR operations and conjugate positions in conjugate groups keep values 0 under AND operations that make two integrated feature vectors be equally valued.

A useful sample case is shown in Figure 1 to illustrate relevant partitions and projections on feature vectors.

Fig. 1 Sample Cases for Feature States and Conjugate Feature Vectors

$m=1, n=2, N=16$ one configuration $X=1011100011001001$ partitions to 4 feature vectors and 2 constant vectors,

$$
\begin{aligned}
\Gamma_{1} & =\{11\}, \Gamma_{2}=\{10\}, \text { two feature states for } 1 \text { values } \\
\tilde{\Gamma}_{1} & =\{00\}, \tilde{\Gamma}_{2}=\{01\}, \text { two conjugate feature states for } 0 \text { values } \\
X & =1011100011001001, \text { one configuration with } 16 \text { bits as a cycle } \\
\neg X & =0100011100110110, \text { reversed configuration } \\
\Gamma_{\emptyset}(X) & =0000000000000000, \overrightarrow{0} \text { constant vector of } \Gamma_{\emptyset} \\
\Gamma_{1}(X) & =0011000010000001, \text { feature vector of } \Gamma_{1} \\
\Gamma_{2}(X) & =1000100001001000, \text { feature vector of } \Gamma_{2} \\
\tilde{\Gamma}_{\emptyset}(X) & =1111111111111111, \overrightarrow{1} \text { conjugate constant vector of } \tilde{\Gamma}_{\emptyset} \\
\tilde{\Gamma}_{1}(X) & =1111100111011011, \text { conjugate feature vector of } \tilde{\Gamma}_{1} \\
\tilde{\Gamma}_{2}(X) & =1011111011101101, \text { conjugate feature vector of } \tilde{\Gamma}_{2} \\
\Gamma(X)=\tilde{\Gamma}(X)=X & =1011100011001001, \text { two conjugate vectors are equal } \\
a_{\emptyset}(X) & =0, \text { counting } 1 \text { values in } \overrightarrow{0} \\
a_{1}(X) & =4 \\
a_{2}(X) & =4 \\
a_{I}(X)=a_{1}+a_{2} & =8, \text { total number of } 1 \text { values in } \Gamma(X) \\
b_{\emptyset}(X) & =0, \text { counting } 0 \text { values in } \overrightarrow{1} \\
b_{1}(X) & =4 \\
b_{2}(X) & =4 \\
b_{I}(X)=b_{1}+b_{2} & =8, \text { total number of } 0 \text { values in } \tilde{\Gamma}(X) \\
N=a_{I}+b_{I} & =16
\end{aligned}
$$




\subsection{Special Logic Operators}

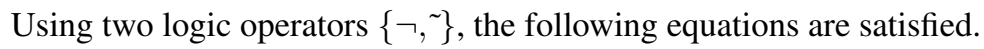

Theorem 3. For two feature vectors $\left\{\Gamma_{j}(X), \tilde{\Gamma}_{j}(X)\right\}$, conjugate operator $\sim$ reverse feature vector and original configuration.

$$
\begin{aligned}
\tilde{\Gamma}_{j}(X) & =\neg \Gamma_{j}(\neg X) ; \\
\neg \tilde{\Gamma}_{j}(X) & =\Gamma_{j}(\neg X) ; \\
\Gamma_{j}(X) & =\neg \tilde{\Gamma}_{j}(\neg X)=\tilde{\Gamma}_{j}(X) ; \\
\neg \Gamma_{j}(X) & =\tilde{\Gamma}_{j}(\neg X)
\end{aligned}
$$

Proof. For the first equation, the $l$-th 0 element of $\tilde{\Gamma}_{j}(X)$ describes the $j$-th group of $X_{l}=\tilde{S}\left(\overrightarrow{y^{l}}\right) \in \tilde{\Gamma}_{j}$, conjugate operator $\neg X_{l}=\tilde{\tilde{S}}\left(\overrightarrow{y^{l}}\right)=\left(1, x_{1}^{l}, \cdots, x_{k}^{l}, \cdots, x_{m}^{l}\right)=$ $S\left(\overrightarrow{y^{l}}\right), x_{k}^{l}=\neg y_{k}^{l}, \tilde{S}\left(\overrightarrow{y^{l}}\right) \in \tilde{\Gamma}_{j} \rightarrow \neg X_{l} \in \Gamma_{j}$. One 1-element of $\Gamma_{j}(\neg X)$ corresponds to one 0 -element of $\tilde{\Gamma}_{j}(X)$ undertaken $\neg$ operations. For other equations, similar operations can be performed in the proof procedure.

\subsection{Irreducible Expression}

When two feature vectors are on a logic expression, if the expression cannot be reduced as a constant vector or simplified as a single feature vector, then this logic expression is an irreducible expression; otherwise, it is a reducible expression.

Corollary 1. For two distinguished feature vectors under $\{\neg, \cap, \cup, \sim\}$ operators, there are only 6 groups of irreducible expressions.

$$
\begin{aligned}
\neg \Gamma_{j}(X) \cap \neg \Gamma_{k}(X) & =\tilde{\Gamma}_{j}(\neg X) \cap \tilde{\Gamma}_{k}(\neg X) ; \\
\Gamma_{j}(X) \cup \Gamma_{k}(X) & =\neg \tilde{\Gamma}_{j}(\neg X) \cup \neg \tilde{\Gamma}_{k}(\neg X) ; \\
\neg \tilde{\Gamma}_{j}(X) \cup \neg \tilde{\Gamma}_{k}(X) & =\Gamma_{j}(\neg X) \cup \Gamma_{k}(\neg X) ; \\
\tilde{\Gamma}_{j}(X) \cap \tilde{\Gamma}_{k}(X) & =\neg \Gamma_{j}(\neg X) \cap \neg \Gamma_{k}(\neg X) ; \\
\neg \Gamma_{j}(X) \cap \tilde{\Gamma}_{k}(X) & =\neg \Gamma_{j}(X) \cap \neg \Gamma_{k}(\neg X) ; \\
\Gamma_{j}(X) \cup \neg \tilde{\Gamma}_{k}(X) & =\Gamma_{j}(X) \cup \Gamma_{k}(\neg X)
\end{aligned}
$$

\section{Conjugate Transformation}

For convenient description of a given configuration, a pair of structures can be established. 


\subsection{A Pair of Parameter Sets}

Let $I=\{1, \cdots, n\}$ be an index set of feature vectors, and $A, B \subseteq I$ be two subsets of the index set.

$$
\langle A, B\rangle=\left\{\left\{\Gamma_{j}\right\}_{j \in A},\left\{\tilde{\Gamma}_{k}\right\}_{k \in B}\right\}
$$

In $\langle A, B\rangle$, the first parameter is the index set of the phase space $\Theta$ and the second parameter is the index set of the conjugate phase space $\tilde{\Theta}$.

e.g., if $A=\{2,4\}, B=\{3,4\}$, then $\langle A, B\rangle=\left\{\Gamma_{2}, \Gamma_{4}, \tilde{\Gamma}_{3}, \tilde{\Gamma}_{4}\right\}$.

\subsection{Pair Sets of Feature Vectors}

For any configuration $X,\langle A, B\rangle X$ represents a pair set of feature vectors.

$$
\langle A, B\rangle X=\left\{\left\{\Gamma_{j}(X)\right\}_{j \in A},\left\{\tilde{\Gamma}_{k}(X)\right\}_{k \in B}\right\}
$$

To express two sets of selected feature vectors, let $\Gamma_{A}(X)$ be a merged set of the index set $A$ under OR operations and $\tilde{\Gamma}_{B}(X)$ be a joined set of the index set $B$ of feature vectors under AND operations.

$$
\begin{aligned}
\Gamma_{A}(X) & =\bigcup_{j \in A} \Gamma_{j}(X) \\
\tilde{\Gamma}_{B}(X) & =\bigcap_{k \in B} \tilde{\Gamma}_{k}(X) \\
\langle A, B\rangle X & =\left\{\Gamma_{A}(X), \tilde{\Gamma}_{B}(X)\right\}
\end{aligned}
$$

$\langle A, B\rangle X$ is composed of two feature vectors.

While $A=B=\emptyset$, let $\Gamma_{\emptyset}(X)=\Gamma_{0}(X)=\overrightarrow{0}$ and $\tilde{\Gamma_{\emptyset}}(X)=\tilde{\Gamma}_{0}(X)=\overrightarrow{1}$ be two constant vectors.

\subsection{Three Set Operators}

Let $\left\{{ }^{\prime}, \vee, \wedge\right\}$ be $\{$ complement, union, intersection $\}$ operators of index sets. For an index set $I, A$ a subset and $A^{\prime}$ a complement subset, $A \subset I$ and $A^{\prime} \subset I$ satisfy the following equation.

$$
\begin{aligned}
A \vee A^{\prime} & =I \\
A \wedge A^{\prime} & =\emptyset \\
\emptyset^{\prime} & =I \\
I^{\prime} & =\emptyset
\end{aligned}
$$


Applying this expression, a canonical vector $\Gamma(X)$ or $\tilde{\Gamma}(X)$ can be described as two parts linked with $\mathrm{a} \cup$ or $\cap$ operator.

$$
\begin{aligned}
& \Gamma(X)=\bigcup_{j=0}^{n} \Gamma_{j}(X)=\bigcup_{j=1}^{n} \Gamma_{j}(X)=\Gamma_{A}(X) \cup \Gamma_{A^{\prime}}(X) \\
& \tilde{\Gamma}(X)=\bigcap_{k=0}^{n} \tilde{\Gamma_{k}}(X)=\bigcap_{k=1}^{n} \tilde{\Gamma}_{k}(X)=\tilde{\Gamma_{B}}(X) \cap \tilde{\Gamma_{B^{\prime}}}(X)
\end{aligned}
$$

\subsection{Basic Operators of Conjugate Transformation}

Using pairs of feature vectors $\langle A, B\rangle X=\left\{\Gamma_{A}(X), \tilde{\Gamma}_{B}(X)\right\}$, it is essential to investigate eight representatives under three logic operators $\{\neg, \cap, \cup\}$. Two irreducible expressions are expressed as $\left\{F_{0}(\langle A, B\rangle X), F_{1}(\langle A, B\rangle X)\right\}$.

Lemma 2. For $A \neq B, \Gamma_{A}(X), \tilde{\Gamma}_{B}(X)$ under $\{\neg, \cap, \cup\}$ operators, there are eight equations.

$$
\begin{aligned}
\Gamma_{A}(X) \cap \tilde{\Gamma}_{B}(X) & =\Gamma_{A}(X) \\
& \Gamma_{A}(X) \cup \tilde{\Gamma_{B}}(X)=\tilde{\Gamma}_{B}(X) \\
F_{0}(\langle A, B\rangle X)=\neg \Gamma_{A}(X) \cap \tilde{\Gamma}_{B}(X) & =\Gamma_{A^{\prime}}(X) \cup \neg \tilde{\Gamma}_{B^{\prime}}(X) \\
\neg \Gamma_{A}(X) \cup \tilde{\Gamma}_{B}(X) & =\overrightarrow{1} \\
\Gamma_{A}(X) \cap \neg \tilde{\Gamma}_{B}(X) & =\overrightarrow{0} \\
F_{1}(\langle A, B\rangle X)= & \Gamma_{A}(X) \cup \neg \tilde{\Gamma}_{B}(X)=\neg \Gamma_{A^{\prime}}(X) \cap \tilde{\Gamma}_{B^{\prime}}(X) \\
\neg \Gamma_{A}(X) \cap \neg \tilde{\Gamma}_{B}(X) & =\neg \tilde{\Gamma}_{B}(X) \\
\neg \Gamma_{A}(X) \cup \neg \tilde{\Gamma}_{B}(X) & =\neg \Gamma_{A}(X)
\end{aligned}
$$

Proof. For Equation (47), feature vector $\Gamma_{A}(X)$ contains positions of states in set $A$ with the value 1 and other positions with value 0 ; and positions of $\tilde{\Gamma}_{B}(X)$ in set $B$ are in values 0 and other positions are in values 1 . Two vectors are operated as two bit vectors, and the result vector has minimal values equal to $\Gamma_{A}(X)$.

For Equation (49): $\neg \Gamma_{A}(X) \cap \tilde{\Gamma}_{B}(X)=\Gamma_{A^{\prime}}(X) \cup \neg \tilde{\Gamma}_{B^{\prime}}(X)$, 


$$
\begin{aligned}
\neg \Gamma_{A}(X) \cap \tilde{\Gamma}_{B}(X)= & \neg \Gamma_{A}(X) \cap \overrightarrow{1} \cap \tilde{\Gamma}_{B}(X) \\
& \because \overrightarrow{1}=\neg \Gamma_{A^{\prime}}(X) \cup \Gamma_{A^{\prime}}(X) \\
= & \neg \Gamma_{A}(X) \cap\left(\neg \Gamma_{A^{\prime}}(X) \cup \Gamma_{A^{\prime}}(X)\right) \cap \tilde{\Gamma}_{B}(X) \\
= & \left(\neg \Gamma_{A}(X) \cap \neg \Gamma_{A^{\prime}}(X) \cup \neg \Gamma_{A}(X) \cap \Gamma_{A^{\prime}}(X)\right) \cap \tilde{\Gamma}_{B}(X) \\
& \because \neg \Gamma_{A}(X) \cap \neg \Gamma_{A^{\prime}}(X)=\neg \Gamma(X), \neg \Gamma_{A}(X) \cap \Gamma_{A^{\prime}}(X)=\Gamma_{A^{\prime}}(X) \\
= & \left(\neg \Gamma(X) \cup \Gamma_{A^{\prime}}(X)\right) \cap \tilde{\Gamma}_{B}(X) \\
& \because \Gamma(X)=\tilde{\Gamma}(X) \\
= & \left(\neg \tilde{\Gamma}(X) \cup \Gamma_{A^{\prime}}(X)\right) \cap \tilde{\Gamma}_{B}(X) \\
= & \neg \tilde{\Gamma}(X) \cap \tilde{\Gamma}_{B}(X) \cup \Gamma_{A^{\prime}}(X) \cap \tilde{\Gamma}_{B}(X) \\
& \because \tilde{\Gamma}(X)=\tilde{\Gamma}_{B}(X) \cap \tilde{\Gamma}_{B^{\prime}}(X), \Gamma_{A^{\prime}}(X) \cap \tilde{\Gamma}_{B}(X)=\Gamma_{A^{\prime}}(X) \\
= & \neg\left(\tilde{\Gamma}_{B}(X) \cap \tilde{\Gamma}_{B^{\prime}}(X)\right) \cap \tilde{\Gamma}_{B}(X) \cup \Gamma_{A^{\prime}}(X) \\
= & \left(\left(\neg \tilde{\Gamma}_{B}(X) \cup \neg \tilde{\Gamma}_{B^{\prime}}(X)\right) \cap \tilde{\Gamma}_{B}(X)\right) \cup \Gamma_{A^{\prime}}(X) \\
= & \left(\left(\neg \tilde{\Gamma}_{B}(X) \cap \tilde{\Gamma}_{B}(X) \cup \neg \tilde{\Gamma}_{B^{\prime}}(X) \cap \tilde{\Gamma}_{B}(X)\right) \cup \Gamma_{A^{\prime}}(X)\right. \\
& \because \neg \tilde{\Gamma}_{B}(X) \cap \tilde{\Gamma}_{B}(X)=\overrightarrow{0}, \neg \tilde{\Gamma}_{B^{\prime}}(X) \cap \tilde{\Gamma}_{B}(X)=\neg \tilde{\Gamma}_{B^{\prime}}(X) \\
= & \left(\left(\overrightarrow{0} \cup \neg \tilde{\Gamma}_{B^{\prime}}(X)\right) \cup \Gamma_{A^{\prime}}(X)\right. \\
= & \neg \tilde{\Gamma}_{B^{\prime}}(X) \cup \tilde{\Gamma}_{A^{\prime}}(X) \\
= & \Gamma_{A^{\prime}}(X) \cup \neg \tilde{\Gamma}_{B^{\prime}}(X) \\
&
\end{aligned}
$$

Following similar patterns, other equations can be proved.

Lemma 3. In general, pairs of functions $F_{0}$ and $F_{1}$ satisfy the following equautions.

$$
\begin{aligned}
& F_{0}(\langle A, B\rangle X)=\neg F_{1}(\langle A, B\rangle X)=F_{1}\left(\left\langle A^{\prime}, B^{\prime}\right\rangle X\right) \\
& F_{1}(\langle A, B\rangle X)=\neg F_{0}(\langle A, B\rangle X)=F_{0}\left(\left\langle A^{\prime}, B^{\prime}\right\rangle X\right)
\end{aligned}
$$

Proof. First, we prove $F_{0}(\langle A, B\rangle X)=\neg F_{1}(\langle A, B\rangle X)$,

$$
\begin{aligned}
\neg F_{1}(\langle A, B\rangle X) & =\neg\left(\Gamma_{A}(X) \cup \neg \tilde{\Gamma}_{B}(X)\right) \\
& =\neg \Gamma_{A}(X) \cap \neg \neg \tilde{\Gamma}_{B}(X) \\
& =\neg \Gamma_{A}(X) \cap \tilde{\Gamma}_{B}(X) \\
& =F_{0}(\langle A, B\rangle X)
\end{aligned}
$$

Then proof $\neg F_{1}(\langle A, B\rangle X)=F_{1}\left(\left\langle A^{\prime}, B^{\prime}\right\rangle X\right)$,

$$
\begin{aligned}
\neg F_{1}(\langle A, B\rangle X)= & \neg \Gamma_{A}(X) \cap \tilde{\Gamma}_{B}(X) \\
& \because \text { Equation }(49) \\
= & \Gamma_{A^{\prime}}(X) \cup \neg \tilde{\Gamma}_{B^{\prime}}(X) \\
= & F_{1}\left(\left\langle A^{\prime}, B^{\prime}\right\rangle X\right)
\end{aligned}
$$


Corollary 2. Selecting functions $F_{0}(\langle A, B\rangle X)$ or $F_{1}(\langle A, B\rangle X)$, the pair of feature vectors of conjugate transformation generates four extreme vectors $\{\Gamma(X), \overrightarrow{0}, \overrightarrow{1}, \neg \Gamma(X)\}$; four extreme vectors are $\langle A, B\rangle=\{\langle\emptyset, \emptyset\rangle,\langle\emptyset, I\rangle,\langle I, \emptyset\rangle,\langle I, I\rangle\}$.

Proof.

$$
\begin{aligned}
F_{0}(\langle\emptyset, \emptyset\rangle X) & =\neg \Gamma_{\emptyset}(X) \cap \tilde{\Gamma}_{\emptyset}(X) \\
& =\neg \overrightarrow{0} \cap \overrightarrow{1} \\
& =\overrightarrow{1} \rightarrow \text { A Constant } 1 \text { Vector } \\
F_{0}(\langle\emptyset, I\rangle X) & =\neg \Gamma_{\emptyset}(X) \cap \tilde{\Gamma}_{I}(X) \\
& =\neg \overrightarrow{0} \cap \Gamma(X) \\
& =\Gamma(X) \rightarrow \text { An Invariant Vector } \\
F_{0}(\langle I, \emptyset\rangle X) & =\neg \Gamma_{I}(X) \cap \tilde{\Gamma}_{\emptyset}(X) \\
& =\neg \Gamma(X) \cap \overrightarrow{1} \\
& =\neg \Gamma(X) \rightarrow \text { A Reversed Vector } \\
F_{0}(\langle I, I\rangle X) & =\neg \Gamma_{I}(X) \cap \tilde{\Gamma}_{I}(X) \\
& =\neg \Gamma(X) \cap \Gamma(X) \\
& =\overrightarrow{0} \mathrm{~A} \text { Constatnt } 0 \text { Vector } \\
F_{1}(\langle\emptyset, \emptyset\rangle X) & =\Gamma_{\emptyset}(X) \cup \neg \tilde{\Gamma}_{\emptyset}(X) \\
& =\overrightarrow{0} \cup \neg \overrightarrow{1} \\
& =\overrightarrow{0} \rightarrow \mathrm{A} \text { Constant } 0 \text { Vector } \\
F_{1}(\langle\emptyset, I\rangle X) & =\Gamma_{\emptyset}(X) \cup \neg \tilde{\Gamma}_{I}(X) \\
& =\overrightarrow{0} \cup \neg \Gamma X) \\
& =\neg \Gamma(X) \rightarrow \text { An Invariant Vector } \\
F_{1}(\langle I, I\rangle X) & =\Gamma_{I}(X) \cup \neg \tilde{\Gamma} I(X) \\
& =\Gamma(X) \cup \neg \Gamma(X) \\
& =\overrightarrow{1} \mathrm{~A} \text { Constatnt } 1 \text { Vector } \\
F_{1}(\langle I, \emptyset\rangle X) & =\Gamma_{I}(X) \cup \neg \tilde{\Gamma_{\emptyset}}(X) \\
& =\Gamma(X) \cup \neg \overrightarrow{1} \\
& =\Gamma(X) \rightarrow \text { A Reversed Vector } \\
&
\end{aligned}
$$

For another set of equations, the results can be verified directly.

From this set of the four results, pair sets of feature vectors are represented by two equivalent equations. One type of equation uses $\cap$ operators, and another uses $\cup$ operators. Choosing any equation, it is possible to cover $[\overrightarrow{0}, \overrightarrow{1}]$ regions on $2^{2 n}$ functions. From quantitative analysis viewpoint, this type of bi-expression cannot be observed in classical logic systems with intrinsic complementarity properties. 
Corollary 3. For a configuration $X$ and its two sets offeature vectors $\left\{\Gamma_{j}(X)\right\}_{j=0}^{n},\left\{\tilde{\Gamma}_{k}(X)\right\}_{k=0}^{n}$, the elementary equation of conjugate transformation can generate $2^{2 n}$ vector functions.

Proof. Each selection of $\langle A, B\rangle$ determines a vector logic function. Two sets are composed of all possible combinations of $A, B \subseteq I=\{1, \cdots, n\}$, each index set has $2^{n}$ selections, and a total of $2^{2 n}$ vector logic functions can be generated.

\subsection{Elementary Equation of Conjugate Transformation}

Using $\langle A, B\rangle X,\left\{F_{0}, F_{1}\right\}$, it is feasible to generate all $2^{2 n}$ vector logic functions from $2 n$ feature vectors. From a symmetric viewpoint, each expression is a reversed form of another one. Since there are internal unbalanced properties, neither equations has self-conjugate symmetry.

From a conjugate symmetric viewpoint, the elementary equation provides better properties in optimal forms for balanced organizations.

Let $\downarrow$ be a reversing operator, it reverses selected parts of the configuration to satisfy the following equations.

Let $F(\langle A, B\rangle X)$ be an elementary equation of Conjugate Transformation

$$
\begin{aligned}
F(\langle A, B\rangle X) & =\Gamma(X) \downarrow\langle A, B\rangle X \\
& =\left(\Gamma(X) \cap\left(\neg \Gamma_{A}(X)\right)\right) \cup\left(\neg \tilde{\Gamma}_{B}(X)\right)
\end{aligned}
$$

Operator $\sharp$ has the following meaning: selected components $\langle A, B\rangle X$, the operator changes selected sets of feature vectors and retains other unselected sets of feature vectors be invariant.

Theorem 4. The elementary equation is a vector logic equation to reverse the selected sets of feature vectors and keep other unselected sets of feature vectors invariant.

Corollary 4. Under the $\neg$ operator, the elementary equation is a self-conjugate equation to exchange index sets of feature vectors and to be reversed on the configuration vector.

$$
\neg F(\langle A, B\rangle X)=F(\langle B, A\rangle \neg X)
$$

Proof. 


$$
\begin{aligned}
\neg F(\langle A, B\rangle X)= & \neg\left(\left(\Gamma(X) \cap\left(\neg \Gamma_{A}(X)\right)\right) \cup\left(\neg \tilde{\Gamma}_{B}(X)\right)\right) \\
& \text { de Morgan Rule } \\
= & \neg\left(\Gamma(X) \cap\left(\neg \Gamma_{A}(X)\right)\right) \cap \tilde{\Gamma}_{B}(X) \\
& \text { Distribution Rule } \\
= & \left(\neg \Gamma(X) \cup \Gamma_{A}(X)\right) \cap \tilde{\Gamma}_{B}(X) \\
& \because \Gamma_{A}(X) \cap \tilde{\Gamma}_{B}(X)=\Gamma_{A}(X) \\
= & \left(\neg \Gamma(X) \cap \tilde{\Gamma}_{B}(X)\right) \cup\left(\Gamma_{A}(X) \cap \tilde{\Gamma}_{B}(X)\right) \\
& \because \tilde{\Gamma}_{B}(X)=\neg \Gamma_{B}(\neg X), \Gamma_{A}(X)=\neg \tilde{\Gamma}_{A}(\neg X) \\
= & \left(\neg \Gamma(X) \cap \tilde{\Gamma}_{B}(X)\right) \cup \Gamma_{A}(X) \\
& \because \neg \Gamma(X)=\tilde{\Gamma}(\neg X)=\Gamma(\neg X) \\
= & \left(\Gamma(\neg X) \cap \neg \Gamma_{B}(\neg X)\right) \cup \neg \tilde{\Gamma}_{A}(\neg X) \\
= & F(\langle B, A\rangle \neg X)
\end{aligned}
$$

Theorem 5. For a $\langle A, B\rangle X \in C T S(X)$, the elementary equation of CTS has four equivalent expressions.

$$
\begin{aligned}
F(\langle A, B\rangle X) & =\left(\Gamma(X) \cap \neg \Gamma_{A}(X)\right) \cup \neg \tilde{\Gamma_{B}}(X) \\
& \left.=\Gamma_{A^{\prime}}(X)\right) \cup \neg \tilde{\Gamma_{B}}(X) \\
& \left.=\neg \Gamma_{A}(X)\right) \cap \tilde{\Gamma_{B^{\prime}}}(X) \\
& =\left(\Gamma(X) \cup \neg \tilde{\Gamma_{B}}(X)\right) \cap \neg \Gamma_{A}(X)
\end{aligned}
$$

Proof. From the first one to the second one, we have

$$
\begin{aligned}
F(\langle A, B\rangle X) & =\left(\Gamma(X) \cap \neg \Gamma_{A}(X)\right) \cup \neg \tilde{\Gamma}_{B}(X) \\
& =\left(\left(\Gamma_{A}(X) \cup \Gamma_{A^{\prime}}(X)\right) \cap \neg \Gamma_{A}(X)\right) \cup \neg \tilde{\Gamma}_{B}(X) \\
& =\left(\Gamma_{A}(X) \cap \neg \Gamma_{A}(X)\right) \cup\left(\Gamma_{A^{\prime}}(X) \cap \neg \Gamma_{A}(X)\right) \cup \neg \tilde{\Gamma_{B}}(X) \\
& =(\overrightarrow{0}) \cup\left(\Gamma_{A^{\prime}}(X) \cap \neg \Gamma_{A}(X)\right) \cup \neg \tilde{\Gamma}_{B}(X) \\
& =\left(\Gamma_{A^{\prime}}(X) \cap \neg \Gamma_{A}(X)\right) \cup \neg \tilde{\Gamma}_{B}(X) \\
& =\Gamma_{A^{\prime}}(X) \cup \neg \tilde{\Gamma}_{B}(X)
\end{aligned}
$$

The other two expressions can be deduced in a similar way.

From the four equivalent expressions, symmetric properties can be observed in the formula.

Corollary 5. For a given $\langle A, B\rangle$, if $A=\emptyset$ or $B=\emptyset$, then $F(\langle A, B\rangle X)$ for components $\langle A, B\rangle X$ is an equation of dilation or erosion.

\section{Proof.}




$$
\begin{aligned}
F(\langle\emptyset, B\rangle X) & =\left(\Gamma(X) \cap\left(\neg \Gamma_{0}(X)\right)\right) \cup\left(\neg \tilde{\Gamma}_{B}(X)\right) \\
& =\Gamma(X) \cup\left(\neg \tilde{\Gamma}_{B}(X)\right)(\text { Dilation }) \\
F(\langle A, \emptyset\rangle X) & =\left(\Gamma(X) \cap\left(\neg \Gamma_{A}(X)\right)\right) \cup\left(\neg \tilde{\Gamma}_{0}(X)\right) \\
& =\Gamma(X) \cap\left(\neg \Gamma_{A}(X)\right) \text { (Erosion) }
\end{aligned}
$$

Corollary 6. The elementary equation of conjugate transformation generates four extreme vectors $\{\Gamma(X), \overrightarrow{0}, \overrightarrow{1}, \neg \Gamma(X)\}$; four extreme vectors are $\langle A, B\rangle=\{\langle\emptyset, \emptyset\rangle$, $\langle\emptyset, I\rangle,\langle I, \emptyset\rangle,\langle I, I\rangle\}$.

Proof.

$$
\begin{aligned}
F(\langle\emptyset, \emptyset\rangle X) & =\left(\Gamma(X) \cap\left(\neg \Gamma_{0}(X)\right)\right) \cup\left(\neg \tilde{\Gamma}_{0}(X)\right) \\
& =\Gamma(X) \rightarrow \text { An Invariant Vector } \\
F(\langle\emptyset, I\rangle X) & =\left(\Gamma(X) \cap\left(\neg \Gamma_{0}(X)\right)\right) \cup\left(\neg \tilde{\Gamma}_{I}(X)\right) \\
& =\Gamma(X) \cup \neg \tilde{\Gamma}_{I}(X) \\
& =\Gamma(X) \cup \neg \tilde{\Gamma}(X) \\
& =\Gamma(X) \cup \neg \Gamma(X) \\
& =\overrightarrow{1} \rightarrow \text { A Constant } 1 \text { Vector } \\
F(\langle I, \emptyset\rangle X) & =\left(\Gamma(X) \cap\left(\neg \Gamma_{I}(X)\right)\right) \cup\left(\neg \tilde{\Gamma}_{0}(X)\right) \\
& =\Gamma(X) \cap\left(\neg \Gamma_{I}(X)\right) \\
& =\Gamma(X) \cap(\neg \Gamma(X)) \\
& =\overrightarrow{0} \rightarrow \text { A Constatnt } 0 \text { Vector } \\
F(\langle I, I\rangle X) & =\left(\Gamma(X) \cap\left(\neg \Gamma_{I}(X)\right)\right) \cup\left(\neg \tilde{\Gamma}_{I}(X)\right) \\
& =(\Gamma(X) \cap(\neg \Gamma(X))) \cup(\neg \tilde{\Gamma}(X)) \\
& =\neg \tilde{\Gamma}(X) \\
& =\neg \Gamma(X) \rightarrow \text { A Reversed Vector }
\end{aligned}
$$

Corollary 7. For a configuration $X$ and its two sets of feature vectors $\left\{\Gamma_{j}(X)\right\}_{j=0}^{n}$, $\left\{\tilde{\Gamma}_{k}(X)\right\}_{k=0}^{n}$, the elementary equation of conjugate transformation can generate $2^{2 n}$ vector functions.

Proof. Each selection of $\langle A, B\rangle$ determines a vector logic function. Two sets are composed of all possible combinations of $A, B \subseteq I=\{1, \cdots, n\}$, each index set has $2^{n}$ selections, and a total of $2^{2 n}$ vector logic functions can be generated. 


\section{Measurement Structure on Complementarity Expression}

\subsection{Pairs of Opposites}

In Section 6, for any configuration $X$, it is feasible to partition $2 n$ clusters of states generating $2 n$ feature vectors in arbitrary combinations. Two basic equations $\left\{F_{0}, F_{1}\right\}$ and the elementary equation $F$, three equations provide full capacity to apply $2 n$ feature vectors to generate all $2^{2 n}$ vector logic functions.

Lemma 4. For any two index sets, the following equations are satisfied.

$$
\begin{aligned}
& A^{\prime} \vee B^{\prime}=(A \wedge B)^{\prime} \\
& (A \vee B)^{\prime}=A^{\prime} \wedge B^{\prime}
\end{aligned}
$$

e.g.

$$
\begin{aligned}
I & =\{1,2,3,4,5,6\} \\
A & =\{1,2,3\}, A^{\prime}=\{4,5,6\} \\
B & =\{3,4\}, B^{\prime}=\{1,2,5,6\} \\
A \vee B & =\{1,2,3,4\}, A \wedge B=\{3\} \\
A^{\prime} \vee B^{\prime} & =(A \wedge B)^{\prime}=\{1,2,4,5,6\} \\
(A \vee B)^{\prime} & =A^{\prime} \wedge B^{\prime}=\{5,6\}
\end{aligned}
$$

Since Conjugate Transformation manages feature vectors as two conjugate sets, let the selected set be $A$ and its complementary set be $A^{\prime}$. From a representative viewpoint, the whole set of feature vectors is in $I$, which makes conjugate mapping into bilinear structures. For a complementarity formalism, this set of expressions provides idea complementary properties to make pairs of opposites on selected and non-selected sets corresponding to local and nonlocal effects, respectively. This paradox style consistently provides the foundational support on both logic and measurement levels.

$$
\begin{aligned}
& A \vee A^{\prime}=I, A \wedge A^{\prime}=\emptyset \\
& B \vee B^{\prime}=I, B \wedge B^{\prime}=\emptyset
\end{aligned}
$$

Using feature vectors and their combinations, all measurements are distributed in the $[0, N]$ region. For a configuration with $N$ length, $2 n$ feature vectors, each vector set $A$ corresponds to $\Gamma_{A}(X) \rightarrow \alpha_{A}$ measurement and its complementary set $A^{\prime}$ corresponds to $\Gamma_{A}^{\prime}(X) \rightarrow \alpha_{A^{\prime}}$.

Considering $0-1$ Logic variables transfer to rational numbers under $\neg$ operators, it is natural to assign a pair of measurements in equal quality and opposite directions satisfing the following equation.

$$
\alpha_{A}=-\alpha_{A^{\prime}}
$$


Under this type of pair, $4 n$ measurements are determined. For $1 \leq i \leq n$, four classes $\left\{A, A^{\prime}, B, B^{\prime}\right\}$ are described as follows.

$$
\begin{aligned}
\left\{a_{1}, \cdots, a_{i}, \cdots, a_{n}\right\} & \rightarrow \text { Class } A \\
\left\{-a_{1}, \cdots,-a_{i}, \cdots,-a_{n}\right\} & \rightarrow \text { Class } A^{\prime} \\
\left\{b_{1}, \cdots, b_{i}, \cdots, b_{n}\right\} & \rightarrow \text { Class } B \\
\left\{-b_{1}, \cdots,-b_{i}, \cdots,-b_{n}\right\} & \rightarrow \text { Class } B^{\prime}
\end{aligned}
$$

\subsection{Probability Space}

Corresponding to probability measurements $\left\{p_{i}, p_{i^{\prime}}, \tilde{p}_{i}, \tilde{p}_{i^{\prime}}\right\}_{i=1}^{n}$ as follows to transfer each class being probability.

$$
\begin{aligned}
& p_{i}=\frac{a_{i}}{a_{I}}, \sum_{i=1}^{n} p_{i}=1 \\
& p_{i^{\prime}}=\frac{a_{i^{\prime}}}{a_{I^{\prime}}}, \sum_{i=1}^{n} p_{i^{\prime}}=1 \\
& \tilde{p}_{i}=\frac{b_{i}}{b_{I}}, \sum_{i=1}^{n} \tilde{p}_{i}=1 \\
& \tilde{p}_{i^{\prime}}=\frac{b_{i^{\prime}}}{b_{I^{\prime}}}, \sum_{i=1}^{n} \tilde{p}_{i^{\prime}}=1
\end{aligned}
$$

A comprehensive sample case is shown in Figure 2 to illustrate various feature vectors, complementary components, measurements and complementary measurements from a given configuration.

From a dimensional viewpoint, the vector length of conjugate transformation is $N$ based on $2 n$ feature vectors with $N$ length. After the logic structure is transformed into a measurement structure, each vector has an opposite to form a total of $4 n$ measurements in a measurement space.

From $0-1$ vectors to be composed into measurement vectors, thhe kernel form of measurements does not contain multiple symmetric properties superior to the original feature vectors to satisfy such as $\Gamma(X)=\tilde{\Gamma}(X)$ equations.

\section{Conclusion}

Under conjugate pairs of partitions on phase spaces, conjugate clusters and feature vectors can be applied to consistently establish various logic equations including both local and nonlocal variables. Two groups of selected sets and their comple- 
Fig. 2 Sample Case for Feature Vectors and Pairs of Conjugate-Complementary Measurements $m=1, n=2, N=16$ one configuration $X=1011100011001001$ partitions to 4 feature vectors and 2 constant vectors,

$$
\begin{aligned}
& \Gamma_{1}=\{11\}, \Gamma_{2}=\{10\}, \text { two feature states for } 1 \text { values } \\
& \tilde{\Gamma}_{1}=\{00\}, \tilde{\Gamma}_{2}=\{01\} \text {, two conjugate feature states for } 0 \text { values } \\
& X=1011100011001001 \text {, one configuration with } 16 \text { bits as a cycle } \\
& \neg X=0100011100110110 \text {, reversed configuration } \\
& \Gamma_{\emptyset}(X)=0000000000000000, \overrightarrow{0} \text { constant vector of } \Gamma_{\emptyset} \\
& \Gamma_{1}(X)=0011000010000001, \text { feature vector of } \Gamma_{1} \\
& \Gamma_{2}(X)=1000100001001000, \text { feature vector of } \Gamma_{2} \\
& \tilde{\Gamma}_{\emptyset}(X)=1111111111111111, \overrightarrow{1} \text { conjugate constant vector of } \tilde{\Gamma}_{\emptyset} \\
& \tilde{\Gamma}_{1}(X)=1111100111011011 \text {, conjugate feature vector of } \tilde{\Gamma}_{1} \\
& \tilde{\Gamma}_{2}(X)=1011111011101101 \text {, conjugate feature vector of } \tilde{\Gamma}_{2} \\
& \Gamma(X)=\tilde{\Gamma}(X)=X=1011100011001001 \text {, two conjugate vectors are equal } \\
& a_{\emptyset}(X)=0 \text {, counting } 1 \text { values in } \overrightarrow{0} \\
& a_{1}(X)=4 \text {, measurements as a selected set } \\
& a_{1^{\prime}}(X)=-4 \text {, measurements as a complementary set } \\
& a_{2}(X)=4 \\
& a_{2^{\prime}}(X)=-4 \\
& a_{I}(X)=a_{1}+a_{2}=8 \text {, total number of } 1 \text { values in } \Gamma(X) \\
& b_{\emptyset}(X)=0 \text {, counting } 0 \text { values in } \overrightarrow{1} \\
& b_{1}(X)=4 \text {, conjugate measurements as a selected set } \\
& b_{1^{\prime}}(X)=-4 \text {, conjugate measurements as a complementary set } \\
& b_{2}(X)=4 \\
& b_{2^{\prime}}(X)=-4 \\
& b_{I}(X)=b_{1}+b_{2}=8, \text { total number of } 0 \text { values in } \tilde{\Gamma}(X) \\
& N=a_{I}+b_{I}=16 \\
& p_{1}=a_{1} / a_{I}=0.5, \Gamma_{1} \text { probability } \\
& p_{1^{\prime}}=a_{1^{\prime}} / a_{I^{\prime}}=0.5, \Gamma_{1} \text { complementary probability } \\
& p_{2}=a_{2} / a_{I}=0.5, \Gamma_{2} \text { probability } \\
& p_{2^{\prime}}=a_{2^{\prime}} / a_{I^{\prime}}=0.5, \Gamma_{2} \text { complementary probability } \\
& \tilde{p}_{1}=b_{1} / b_{I}=0.5, \tilde{\Gamma}_{1} \text { conjugate probability } \\
& \tilde{p}_{1^{\prime}}=b_{1^{\prime}} / b_{I^{\prime}}=0.5, \tilde{\Gamma}_{1} \text { conjugate-complementary probability } \\
& \tilde{p}_{2}=b_{2} / b_{I}=0.5, \tilde{\Gamma}_{2} \text { conjugate probability } \\
& \tilde{p}_{2^{\prime}}=b_{2^{\prime}} / b_{I^{\prime}}=0.5, \tilde{\Gamma}_{2} \text { conjugate-complementary probability }
\end{aligned}
$$


mentary sets correspond to $4 n$ measurements under measurement operators. From a physical application viewpoint, relevant measurements provide both positive and negative values for their energies. Four meta measurements support four output variables of the Hardy paradox in equations without any intrinsic contradiction.

Because new expressions of local and nonlocal complementarity provide additional information, further explorations are required.

Sample cases on two basic equations and equivalent expressions of elementary equations are merely examples. The conjugate transformation structure to handle transforming equations of Hamilton dynamics can be checked in [37].

Under this unified logic framework, it may be possible to provide solid logic foundations on quantum mechanics, quantum information, quantum gravity, etc. to resolve other paradoxes and mysteries in further explorations.

\section{Conflict Interest}

No conflict of interest has been claimed.

\section{References}

1. Rechenbarch H. Philosophic Foundations of Quantum Mechanics, University of California Press, 1945.

2. F. Wolf. Taking the Quantum Leap. Harper \& Row Publishers 1989

3. A. Einstein, B. Podolsky, N. Rosen. Can Quantum-mechanical Description of Physical Reality be Considered Complete? Phys. Rev. 47 770-780 (1935).

4. N. Bohr, Can Quantum-mechanical Description of Physical Reality be Considered Complete? Phys. Rev. 48 696-702 (1935).

5. D. Bohm, A Suggested Interpretation of Quantum Theory in Terms of Hidden Variables, I and II,Physical Review 85: 166-193 (1952)

6. Y. Aharonov and D. Bohm, Significance of Electromagnetic Potentials in Quantum Theory, Physical Review 115: 485-491 (1959)

7. Y. Aharonov and B. Reznik, Complementarity between Local and Nonlocal Topological Effects, Physical Review Letters 84(21) 4790-4793, 2000.

8. Y. Aharonov, A. Botero, S. Popescu, B. Reznik and J. Tollaksen, Revisiting Hardy's paradox: counterfactual statements, real measurements, entanglement and weak values, Physical Letters A 301(2002) 130-138.

9. Y. Aharonov and L. Vaidman, The Two-State Vector Formalism: An Updated Review, Lecture Notes Physics 734 399-447, Springer-Verlag 2008 DOI 10.1007/978-3-540-73473-4_13

10. Y. Aharonov, T. Kaufherr, S. Nussinov, Some aspect of classical and quantum phases, Carolina International Symposium on Neutrino Physics, Journal of Physics: Conference Series 173(2009) 012020

11. Y. Aharonov, J. Behrndt, F. Colombo, P. Schlosser, Schroedinger evolution of superoscillations with $\delta$ - and $\delta^{\prime}$-potentials, Quantum Studies: Mathematics and Foundations https://doi.org/10.1007/s40509-019-00215-4 2019

12. J.S. Bell, On the Einstein-Podolsky-Rosen paradox. Physics 1, 195 (1964) https://doi.org/10.1103/PhysicsPhysiqueFizika.1.195 
13. J.S. Bell, Speakable and Unspeakable in Quantum Mechanics, Cambridge University Press (1987)

14. J.F. Clauser, M.A. Horne, A. Shimony and R.A. Holt. Proposed experiment to test local hidden-variable theories. Phys. Rev. Lett. 23, 880 (1969)

15. D.M. Greenberger, M.A. Horne, and A. Zeilinger. Bell's Theorem without Inequalities, Am. J. Phys. 58 1131-1143 (1990)

16. D. Bouwmeester, J.W. Pan, M. Daniell, H. Weinfurter and A. Zeilinger, Observation of threephoton Greenberger-Horne-Zeilinger entanglement. Phys. Rev. Lett. 82 1345-1349 (1999)

17. Schumann, R. Quantum Correlations, Nuclearity, and Spacetime Curvature. Letters in Mathematical Physics (1999) 47: 1. https://doi.org/10.1023/A:1007553113988

18. Halvorson, H. The EinsteinPodolskyRosen State Maximally Violates Bell's Inequalities. Letters in Mathematical Physics (2000) 53: 321. https://doi.org/10.1023/A:1007609031556

19. J.W. Pan et al. Multiphoton entanglement and interferometry. Rev.Mod. Phys. 84, 777-838 (2012)

20. Dai Pra, P., Pavon, M. \& Sahasrabudhe, N. A Note on the Geometric Interpretation of Bell ' s Inequalities. Letters in Mathematical Physics 103, 11651170 (2013) doi:10.1007/s11005-0130631-8

21. W. Tang, S. Yu and C. Oh. Multisetting Greenberger-Horne-Zeilinger paradoxes. Phys. Rev. A 95, 012131 (2017).

22. L.Hardy, Quantum mechanics, local realistic theories and Lorentz-invariant realistic theories. Phys. Rev. Lett. 68, 2981 (1992)

23. L. Hardy. Nonlocality for two particles without inequalities for almost all entangled states. Phys. Rev. Lett. 71(11) 1665

24. N.D. Mermin. Quantum Mysteries Refined. Am. J. Phys. 1994:62:880

25. J.L. Cereceda, Hardy's nonlocality for generalized n-particle GHZ states, Physics Letters A $327,433(2004)$

26. J.S. Lundeen and A.M. Steinberg, Experimental Join Weak Measurement on a Photon Pairs as a Probe of Hardy's Paradox, Physical Review Letters 102, 020404 (2009)

27. K. Yokota, T. Yamamotor, M. Koashi and N. Imoto, Direct observation of Hardy's paradox by joint weak measurement with an entangled photon pair, New Journal of Physics 11(2009)033011(9pp)

28. Zhe CHEN, YongJian HAN, Quantum nonlocality, quantum entanglement and new physics, Chinese Science Bulletin 61(10), 1072-1074 (2016) 10.1360/N972016-00123

29. Yi-Han Luo, Hong-Yi Su, He-Liang Huang, Xi-Lin Wang, Tao Yang, Li Li, Nai-Le Liu, JingLing Chen, Chao-Yang Lu, Jian-Wei Pan, Experimental test of generalized Hardy's paradox, Science Bulletin 63(24), 1611-1615 (2018) 10.1016/j.scib.2018.11.025

30. Guilu Long, Physicists experimentally verify the multipartite generalized Hardy's paradox, Science Bulletin 63(24), 1597 (2018) 10.1016/j.scib.2018.12.011

31. HuaiXin Cao, ZhiHua Guo, Characterizing Bell nonlocality and EPR steering, SCIENCE CHINA Physics, Mechanics \& Astronomy 62(3), 030311 (2019) 10.1007/s11433-018-92794

32. Z.J. Zheng and A.J. Maeder, The Conjugate Classification of the Kernel Form of the Hexagonal Grid, in Modern Geometric Computing for Visualization, T.L. Kunii and Y.Shinagawa Eds, Springer-Verlag 73-89, 1992

33. Z.J. Zheng and A.J. Maeder, The Elementary Equation of the Conjugate Transformation for Hexagonal Grid, in Modeling in Computer Graphics, eds by B. Falcidieno and T.L. Kunii, Springer-Verlag 21-42, 1993.

34. Z.J. Zheng and A.J. Maeder, The Reversible Phenomena and the Conjugate Classification, Proceedings of 1993 Australian Cognitive Science Conference, 43-45 1993.

35. Z.J. Zheng, Conjugate Transformation of Regular Plane Lattices for Binary Images, PhD Thesis, Monash University 1994.

36. J. Zheng, Variant construction from Theoretical Foundation to Applications, Springer Nature 2019

37. J. Zheng, Measurement Operators in Conjugate Transformation Structure. 


\section{Figures}

$$
\begin{aligned}
\Gamma_{1} & =\{11\}, \Gamma_{2}=\{10\}, \text { two feature states for } 1 \text { values } \\
\bar{\Gamma}_{1} & =\{00\}, \tilde{\Gamma}_{2}=\{01\}, \text { two conjugate feature states for } 0 \text { values } \\
X & =1011100011001001, \text { one configuration with } 16 \text { bits as a cycle } \\
\neg X & =0100011100110110, \text { reversed configuration } \\
\Gamma_{\emptyset}(X) & =0000000000000000, \overrightarrow{0} \text { constant vector of } \Gamma_{\emptyset} \\
\Gamma_{1}(X) & =0011000010000001, \text { feature vector of } \Gamma_{1} \\
\Gamma_{2}(X) & =1000100001001000, \text { feature vector of } \Gamma_{2} \\
\tilde{\Gamma}_{\emptyset}(X) & =1111111111111111, \overrightarrow{1} \text { conjugate constant vector of } \tilde{\Gamma}_{\emptyset} \\
\tilde{\Gamma}_{1}(X) & =1111100111011011, \text { conjugate feature vector of } \tilde{\Gamma}_{1} \\
\tilde{\Gamma}_{2}(X) & =1011111011101101, \text { conjugate feature vector of } \tilde{\Gamma}_{2} \\
\Gamma(X)=\tilde{\Gamma}(X)=X & =1011100011001001, \text { two conjugate vectors are equal } \\
a_{\emptyset}(X) & =0, \text { counting } 1 \text { values in } \overrightarrow{0} \\
a_{1}(X) & =4 \\
a_{2}(X) & =4 \\
a_{I}(X)=a_{1}+a_{2} & =8, \text { total number of } 1 \text { values in } \Gamma(X) \\
b_{\emptyset}(X) & =0, \text { counting } 0 \text { values in } \overrightarrow{1} \\
b_{1}(X) & =4 \\
b_{2}(X) & =4 \\
N=a_{I}+b_{I} & =16
\end{aligned}
$$

\section{Figure 1}

Sample Cases for Feature States and Conjugate Feature Vectors $m=1, n=2, N=16$ one configuration $X$ $=1011100011001001$ partitions to 4 feature vectors and 2 constant vectors, 


$$
\begin{aligned}
& \Gamma_{1}=\{11\}, \Gamma_{2}=\{10\}, \text { two feature states for } 1 \text { values } \\
& \tilde{\Gamma}_{1}=\{00\}, \bar{\Gamma}_{2}=\{01\} \text {, two conjugate feature states for } 0 \text { values } \\
& X=1011100011001001 \text {, one configuration with } 16 \text { bits as a cycle } \\
& \neg X=0100011100110110 \text {, reversed configuration } \\
& \Gamma_{\emptyset}(X)=0000000000000000, \overrightarrow{0} \text { constant vector of } \Gamma_{\emptyset} \\
& \Gamma_{1}(X)=0011000010000001 \text {, feature vector of } \Gamma_{1} \\
& \Gamma_{2}(X)=1000100001001000 \text {, feature vector of } \Gamma_{2} \\
& \bar{\Gamma}_{\theta}(X)=1111111111111111, \overrightarrow{1} \text { conjugate constant vector of } \tilde{\Gamma}_{\emptyset} \\
& \bar{\Gamma}_{1}(X)=1111100111011011 \text {, conjugate feature vector of } \tilde{\Gamma}_{1} \\
& \bar{\Gamma}_{2}(X)=1011111011101101 \text {, conjugate feature vector of } \bar{\Gamma}_{2} \\
& \Gamma(X)=\tilde{\Gamma}(X)=X=1011100011001001 \text {, two conjugate vectors are equal } \\
& a_{\emptyset}(X)=0 \text {, counting } 1 \text { values in } \overrightarrow{0} \\
& a_{1}(X)=4 \text {, measurements as a selected set } \\
& a_{1^{\prime}}(X)=-4 \text {, measurements as a complementary set } \\
& a_{2}(X)=4 \\
& a_{2^{\prime}}(X)=-4 \\
& a_{I}(X)=a_{1}+a_{2}=8 \text {, total number of } 1 \text { values in } \Gamma(X) \\
& b_{\bullet}(X)=0 \text {, counting } 0 \text { values in } \overrightarrow{1} \\
& b_{1}(X)=4 \text {, conjugate measurements as a selected set } \\
& b_{1^{\prime}}(X)=-4 \text {, conjugate measurements as a complementary set } \\
& b_{2}(X)=4 \\
& b_{2^{\prime}}(X)=-4 \\
& b_{I}(X)=b_{1}+b_{2}=8 \text {, total number of } 0 \text { values in } \breve{\Gamma}(X) \\
& N=a_{I}+b_{I}=16 \\
& p_{1}=a_{1} / a_{I}=0.5, \Gamma_{1} \text { probability } \\
& p_{I^{\prime}}=a_{1^{\prime}} / a_{I^{\prime}}=0.5, \Gamma_{1} \text { complementary probability } \\
& p_{2}=a_{2} / a_{I}=0.5, \Gamma_{2} \text { probability } \\
& p_{2^{\prime}}=a_{2^{\prime}} / a_{l^{\prime}}=0.5, \Gamma_{2} \text { complementary probability } \\
& \bar{p}_{1}=b_{1} / b_{1}=0.5, \tilde{\Gamma}_{1} \text { conjugate probability } \\
& \tilde{p}_{1^{\prime}}=b_{1^{\prime}} / b_{I^{\prime}}=0.5, \tilde{\Gamma}_{1} \text { conjugate-complementary probability } \\
& \bar{p}_{2}=b_{2} / b_{l}=0.5, \bar{\Gamma}_{2} \text { conjugate probability } \\
& \bar{p}_{2^{\prime}}=b_{2^{\prime}} / b_{I^{\prime}}=0.5, \tilde{\Gamma}_{2} \text { conjugate-complementary probability }
\end{aligned}
$$

\section{Figure 2}

Sample Case for Feature Vectors and Pairs of Conjugate-Complementary Measurements $m=1, n=2, N=$ 16 one configuration $X=1011100011001001$ partitions to 4 feature vectors and 2 constant vectors, 